\title{
Quasiparticle spectrum of a type-II superconductor in a high magnetic field with randomly pinned vortices
}

\author{
P.D. Sacramento \\ Departamento de Física and CFIF, Instituto Superior Técnico , Av. Rovisco Pais, P-1096 Lisboa Codex, Portugal
}

\begin{abstract}
We show that gapless superconductivity of a strongly type-II superconductor in a high magnetic field prevails in the presence of disorder, suggesting a topological nature. We calculate the density of states of the Bogoliubov-de Gennes quasi-particles for a two-dimensional inhomogeneous system in both cases of weak and strong disorder. In the limit of very weak disorder, the effect is very small and the density of states is not appreciably changed. As the disorder increases, the density of states at low energies increases and the ratio of the low energy density of states to its maximum increases significantly.
\end{abstract}

The interplay between superconductivity and a magnetic field has attracted interest for a long time. For sufficiently strong fields the Meissner phase is destroyed and a mixed state appears in the form of a quantized vortex latticel. The superconductor order parameter has zeros at the vortex locations, through which the external magnetic field penetrates in the sample. Contrarily to previous understanding, the increase of the magnetic field intensity, and its associated diamagnetic pair breaking, is counteracted at high magnefic fields by the Landau level structure of the electronst. This leads to interesting properties such as enhancement of the superconducting transition temperature at very high magnetic fields where the electrons are confined to the lowest Landau level2. Associated with the zeros of the order parameter in real space are gapless points in the magnetic Brillouin zone3, which lead to qualitatively different behavior at low temperatures and high magnetic fields 1 .

It has been argued that the gapless behavior is restricted to high fields very close to the upper critical line where the so-called diagonal approximation (where the coupling between Landau levels is neglected) is valid. It has been shown, however, that the presence of offdiagonal terms does not destroy this behaviort and that a perturbation scheme on the off-diagonal terms is possible, as long as there are no band-crossings 5 . It was shown analytically to all orders in the perturbation theory on the off-diagonal terms that there is always a discrete set of points which are gapless that are associated with coherent propagation of the quasiparticles (so-called Eilenberger points). These nodes are associated with the center of mass coordinates of the Cooper pairs and not to some internal structure like in d-wave superconductors. Lowering the magnetic field, a quantum levelcrossing transition has been found that eventually leads to a gapped regime and to states localized in the vortex cores 6 过.

On the other hand, the effect of disorder on superconductivity has also attracted interest for a long time. In the case of non-magnetic impurities and s-wave pairing Anderson's theorem states that, at least for low concentrations, they haye little effect since the impurities are not pair-breakingl. In d-wave superconductors however, non-magnetic impurities cause a strong pair break- ing effect 1 . In the limit of strong scattering it was found that the lowest energy quasiparticles become localized below the mobility gap, even in a regime where the singleelectron wave-functions are still extended 10 . This result has been confirmed solving the Bogoliubov-de Gennes equations with a finite concentration of non-magnetic impurities 11 . However, allowing for angular dependent impurity scattering potentials it has been found that the scattering processes close to the gap nodes may give rise to extended gapless regions 2 . The case of magnetic impurities in the s-wave case also leads to gapless superconductivity 13 .

The question we wish to address in this paper is if the presence of disorder affects the gapless behavior found in the low- $T$ high magnetic field regime of s-wave superconductors discussed above. The case of a dirty but homogeneous superconductor was considered before 14 . It was assumed that the order parameter is not significantly affected by the impurities and retains its periodic structure. It was found that when the disorder becomes stronger than some critical value, a finite density of states appears at the Fermi surface.

In general, since the interactions between the vortices are repulsive, a lattice structure is more favorable energetically. However, if pinning centers are present in the system, the higher energies of different configurations of the vortices may be offset by the presence of disorder. The question then arises if the gapless behavior prevails in this more general case. If the magnetic field is very high, such that the system is in the quantum limit where the electrons are confined to the lowest Landau level, it has been shown that for an arbitraryconfiguration of zeros there is at least one gapless point 15 . In this paper we will consider a more general case in which the off-diagonal terms are included.

In the mean-field approximation the Hamiltonian of the superconducting system can be diagonalized and the energy eigenvalues are the solutions of the Bogoliubov-de Gennes equations $(\mathrm{BdG}) 16$

$$
\begin{array}{r}
{\left[\frac{1}{2 m}\left(\vec{p}-\frac{e}{c} \vec{A}\right)^{2}-\mu\right] u(\vec{r})+\Delta(\vec{r}) v(\vec{r})=E u(\vec{r})} \\
-\left[\frac{1}{2 m}\left(\vec{p}+\frac{e}{c} \vec{A}\right)^{2}-\mu\right] v(\vec{r})+\Delta^{*}(\vec{r}) u(\vec{r})=E v(\vec{r})
\end{array}
$$


where $\Delta(\vec{r})$ is the order parameter and $E$ the energy. If $\Delta(\vec{r})=0$, the solutions are the Landau eigenfunctions which, for a two-dimensional system perpendicular to the magnetic field, read in the Landau gauge

$$
\phi_{n q}=\frac{1}{\sqrt{L_{x}}} \frac{1}{\sqrt{l \sqrt{\pi} 2^{n} n !}} e^{i q x} e^{-\frac{1}{2}\left[\frac{y}{l}+q l\right]^{2}} H_{n}\left[\frac{y}{l}+q l\right]
$$

where $n$ is the Landau index, $L_{x}$ is the length of the system in the $x$ direction, $l$ is the magnetic length given by $l^{2}=\hbar c / e H$ and $H_{n}$ is an Hermite polynomial. The energy eigenvalues are those of an harmonic oscillator centered at $y_{0}=-q l^{2}$

$$
E_{n}=\hbar \omega_{c}\left(n+\frac{1}{2}\right)
$$

where $\omega_{c}$ is the cyclotron frequency. Taking $L_{y}$ to be the dimension along $y$ we obtain that $-L_{y} / 2 \leq q l^{2} \leq L_{y} / 2$. In the presence of $\Delta(\vec{r}) \neq 0$ we can use the Landau basis like

$$
\begin{aligned}
& u(\vec{r})=\sum_{n q} u_{n q} \phi_{n q}(\vec{r}) \\
& v(\vec{r})=\sum_{n q} v_{n q} \phi_{n q}^{*}(\vec{r})
\end{aligned}
$$

and obtain the corresponding eigensystem

$$
\begin{array}{r}
{\left[n-n_{c}\right] u_{n k}^{\mu}+\sum_{m q} v_{m q}^{\mu} \Delta_{n m}^{k q}=\epsilon^{\mu} u_{n k}^{\mu}} \\
-\left[n-n_{c}\right] v_{n k}^{\mu}+\sum_{m q} u_{m q}^{\mu}\left(\Delta_{m n}^{q k}\right)^{*}=\epsilon^{\mu} v_{n k}^{\mu}
\end{array}
$$

where $\epsilon^{\mu}=E^{\mu} /\left(\hbar \omega_{c}\right), n_{c}$ is defined by $\mu=\hbar \omega_{c}\left(n_{c}+\frac{1}{2}\right)$ and

$$
\Delta_{n m}^{k q}=\int d \vec{r} \phi_{n k}^{*}(\vec{r}) \frac{\Delta(\vec{r})}{\hbar \omega_{c}} \phi_{m q}^{*}(\vec{r})
$$

The excitation spectrum is then obtained solving eqs. (5) with an appropriate choice for the order parameter.

In the lattice case, Abrikosov's solution can be written in the Landau gauge as

$$
\Delta(\vec{r})=\Delta \sum_{p} e^{i \pi \frac{b_{x}}{a} p^{2}} e^{i \frac{2 \pi p}{a} x} e^{-\left(\frac{y}{l}+\frac{\pi p}{a} l\right)^{2}}
$$

The vortex lattice is characterized by unit vectors $\vec{a}=$ $(a, 0)$ and $\vec{b}=\left(b_{x}, b_{y}\right)$ where $b_{x}=0, b_{y}=a$ for a square lattice and $b_{x}=\frac{1}{2} a, b_{y}=\frac{\sqrt{3}}{2} a$ for a triangular lattice. This form for the order parameter is valid sufficiently close to the upper critical field, since it is entirely contained in the lowest Landau level of Cooper charge $2 e$. We will not consider contributions to the order parameter from higher Landau levels. In this case the self-consistent equation for the order parameter reduces to a single relation between $\Delta$ and $V$, the attractive interaction strength between the electrons. In the following we will consider the square lattice $\left(L_{x}=L_{y}=L\right)$, for simplicity. In this case the lattice constant $a=l \sqrt{\pi}$ and the zeros are located at the points $x_{i}=\left(i+\frac{1}{2}\right) l \sqrt{\pi}, y_{j}=\left(j+\frac{1}{2}\right) l \sqrt{\pi}$.

An expression for the order parameter has also been found for an arbitrary distribution of the zeros which in the symmetric gauge can be written as 17

$$
\Delta(x, y)=\bar{\Delta} \prod_{i=1}^{N_{\phi}}\left[\frac{x-x_{i}}{l}+i \frac{y-y_{i}}{l}\right] e^{-\frac{1}{2 l^{2} N_{\phi}}\left[\left(x-x_{i}\right)^{2}+\left(y-y_{i}\right)^{2}\right]}
$$

Here, $N_{\phi}$ is the number of vortices in the system (number of zeros $\left.\left(x_{i}, y_{i}\right)\right)$. The solution of the BdG equations is then simply obtained numerically using eq. (6) and performing the gauge transformation of eq. (8) to the Landau gauge.

In the lattice case it is more convenient to use a representation in terms of the magnetic wave-functions 1 , 1 instead of eq. (4), to take advantage of the translational invariance. The generalization to a random configuration is however more conveniently done using a real space representation. We consider a finite system and we have to take account of finite size effects. In particular, the use of eq. (8) for $\Delta(\vec{r})$ is very sensitive. We have therefore compared eqs. (7) and (8) for a finite system. (It is convenient to define new variables $X=\frac{x}{L}, Y=\frac{y}{L}$ and to use that $\left.L=l \sqrt{\pi N_{\phi}}\right)$. The effect of the finite size was eliminated (less than $1 \%$ difference with respect to Abrikosov's solution) using periodic boundary conditions and calculating $\Delta(x, y)$ using eq. (8) over a set of zeros contained in a circle of unit radius (recall that $\left.-\frac{1}{2} \leq X, Y \leq \frac{1}{2}\right)$ and relating $\bar{\Delta}$ with $\Delta$ to give the same amplitude. Having obtained the excitation spectrum and the eigenvectors we calculated the density of states using the expression

$$
\rho(\omega)=\sum_{\mu}\left[\sum_{n q}\left|u_{n q}^{\mu}\right|^{2} \delta\left(\omega-\epsilon^{\mu}\right)+\sum_{n q}\left|v_{n q}^{\mu}\right|^{2} \delta\left(\omega+\epsilon^{\mu}\right)\right]
$$

where the sum is restricted to $\epsilon^{\mu} \geq 0$.

We considered three cases: i) square lattice, ii) weakly disordered lattice and iii) randomly pinned configuration (strong disorder). In the case of weak disorder we considered a distribution of zeros $X_{i}=X_{i}^{L}+\delta\left(-\frac{1}{2}+r\right) / \sqrt{N_{\phi}}$, where $X_{i}^{L}$ is the regular lattice location, $\delta$ is an adjustable amplitude and $r$ is a random number $0<r<1$ (and similarly for $Y_{i}$ ). In the strong disorder case we allow $X_{i}, Y_{i}$ to take any values on the system. We calculated the average over disorder directly on the density of states. That is, we select randomly one configuration of the zeros and 


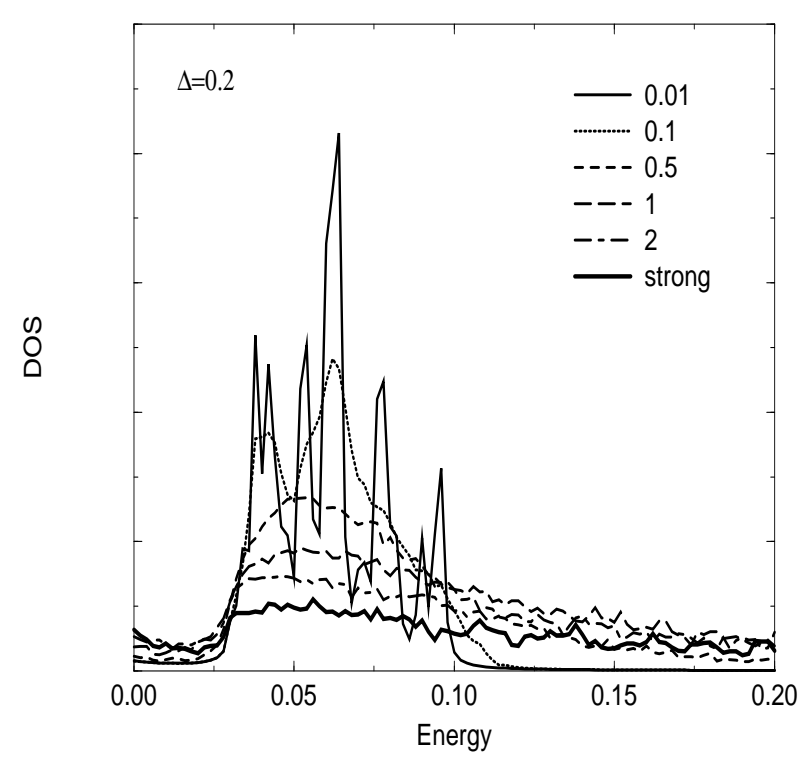

FIG. 1. Density of states (DOS) (in arbitray units) as a function of energy for $n_{c}=10 \pm 2$, for $\Delta=0.2$ for the cases of $\delta=0.01,0.1,0.5,1,2$ and for the case of strong disorder with adjusted chemical potential (see text). Only the lowest band is shown.

obtain the excitation spectrum and $\rho(\omega)$. We repeat this process many times and then we take the average over the resulting expressions for the density of states. This is the final result.

To check the accuracy of the method we started with the lattice case previously solved 6 . The Cooper pairs are formed from electrons in the same point in space and in an energy interval of the order of the Debye energy, which is taken to be typically of the order of $10-20 \%$ of the Fermi energy (therefore we consider off-diagonal couplings between Landau levels with $n_{c}-n_{D} \leq n \leq n_{c}+n_{D}$, where $\left.n_{D} \sim \omega_{D} / \omega_{c}\right)$.

The excitation spectrum depends on $\Delta$ and on the dimensionality of the lattice 0 . It also depends on $n_{c}$ but appropriate rescalings yield a soppewhat universal behavior for not too large values of $\Delta 4.5$. For very small $\Delta$ (in units of the Landau spacing) a diagonal approximation gives good results and a gapless behavior is found at a series of points in the magnetic Brillouin zone associated with the zeros in the real lattice (Eilenberger points) and in other points which increase in number as $n_{c}$ grows. These gapless points contribute to a density of states that vanishes linearly as $\omega \rightarrow 0$. As $\Delta$ grows, off-diagonal terms have to be included. Their effect is two-fold since they affect both the normal self-energy and the pairing self-energy. This leads to a shift in the chemical potential to simulate the $3 \mathrm{D}$ case. In this case it can be shown that the Eilenberger points remain gapless to all orders in the off-diagonal coupling, as long as $\Delta$ is small enough that no band-crossings occur. For $n_{c}$ not too large

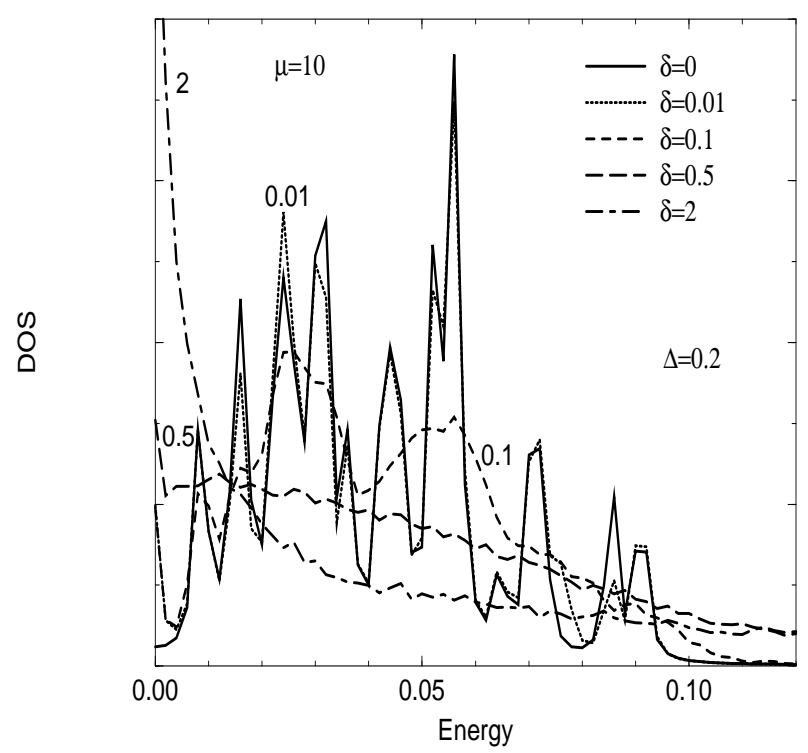

FIG. 2. Density of states (DOS) (in arbitray units) as a function of energy for $n_{c}=10 \pm 2$, and $\mu=10$ for $\Delta=0.2$ for the cases of $\delta=0,0.01,0.1,0.5,1,2$. Only the lowest band is shown.

and with the inclusion of off-diagonal coupling, a pseudogap opens in the spectrum since the weight of the gapless points is small (but nonzero!). (This is peculiar to a $2 \mathrm{~d}$ system since in this case the Fermi surface only passes through a finite number of points, in general. In $3 \mathrm{~d}$ there is always a value of $k_{z}$ such that it is possible to find a gapless point 5 ). As $n_{c}$ grows, and for values of $\Delta$ such that no band-crossings occur, the density of states is more and more similar to the diagonal approximation.

We want to see if the presence of disorder affects this gapless behavior and therefore we consider first the less favorable case where a small density of states is already present in the lattice case. We therefore consider a typical case of $n_{\mathfrak{p}}=10, n_{D}=2$ and $\Delta=0.2$ which was studied before . We maintained the value of the chemical potential fixed and independent of disorder.

In the lattice case the results are very similar using either the magnetic Brillouin formulation or the real space formulation. Due to the finiteness of the system there is somewhat more structure in $\rho(\omega)$ but the qualitative features are the same: a pseudogap of order 0.02 , the location of the maximum around $\omega \sim 0.07$ and the width of order 0.1 for the lowest band above the Fermi level.

Considering now the case of weak disorder we performed averages over 100 configurations for $\delta=$ $0,0.01,0.1,0.5,1,2$. Our results are shown in Fig. 1 . The cases $\delta=0$ and $\delta=0.01$ are virtually indistinguishable. As $\delta$ increases the effect of disorder is clear. For $\delta=0.1$ the width of the band is almost unaltered but the structure is now broadened. For larger values of $\delta$ this effect is more pronounced and the width of the band increases. 
In particular, the density of states for small $\omega$ increases and the relative weight of the gapless modes increases with respect to the maximum $\rho(\omega)$, which remains approximately at the same energy. The case of $\delta=2$ is already very similar to the case of strong disorder where the randomness is maximized. Even though the disorder strongly affects the $u(\vec{r})$ and $v(\vec{r})$ amplitudes we found no evidence for localization. The lowest energy eigenvectors extend considerably over the whole system even though they are strongly inhomogeneous.

The pseudogap of Fig. 1 found in the $2 d$ case for $n_{c}=10$ with the chemical potential adjusted disappears over a wide range of the order parameter if the number of levels increases, Also, as discussed above, in $3 d$ there is always a value of $k_{z}$ such that the pseudogap vanishes. Keeping the chemical potential at $\mu=10$ the pseudogap also becomes small since, even though the Eilenberger points are not gapless in general, as long as the order parameter is small, the gaps are very small throughout the Brillouin zone. In Fig. 2 we consider the case of $n_{c}=10$ and $\mu=10$ as a function of disorder. The case $\delta=0$ shows a small finite density of states at the Fermi level (due to the finite size considered) and a large density of states at low energies. As the disorder increases its effect is very pronounced. The DOS broadens as before and extends from zero energy with a zero energy value that increases as the $\delta$ increses. For strong disorder $(\delta=2)$ the DOS is much larger than the lattice result.

These results show that in the presence of disorder the gapless behavior does not disappear and is actually enhanced. Our numerical results indicate that there is a finite density of states at zero energy particularly in $3 d$ or if the number of Landau levels is not too small. They also confirm that the gapless behavior has a topological nature and is not specific to the periodic vortex lattice.

The author acknowledges helpful discussions with Zlatko Tesanovic and partial support from PRAXIS
Project /2/2.1/FIS/302/94.

${ }^{1}$ A.A. Abrikosov, Sov. Phys. JETP 5, 1174 (1957).

${ }^{2}$ M. Rasolt and Z. Tesanovic, Rev. Mod. Phys. 64, 709 (1992).

${ }^{3}$ S. Dukan, A.V. Andreev and Z. Tesanovic, Physica C 183, 355 (1991).

${ }^{4}$ S. Dukan and Z. Tesanovic, Phys. Rev. B 49, 13017 (1994).

${ }^{5}$ Z. Tesanovic and P.D. Sacramento, Phys. Rev. Lett. 80, 1521 (1998).

${ }^{6}$ M.R. Norman, A.H. MacDonald and H. Akera, Phys. Rev. B 51, 5927 (1995).

${ }^{7}$ C. Caroli, P.G. de Gennes and J. Matricon, Phys. Lett. 9, 307 (1964).

${ }^{8}$ P.W. Anderson, Phys. Rev. Lett. 3, 325 (1959).

${ }^{9}$ K. Ueda and T.M. Rice, in "Theory of Heavy Fermions and Valence Fluctuations", Ed. T. Kasuya and T. Saso (Springer, Berlin, 1985).

${ }^{10}$ P.A. Lee, Phys. Rev. Lett. 71, 1887 (1993); Y. Hatsugai and P.A. Lee, Phys. Rev. B 48, 4204 (1993).

${ }^{11}$ M. Franz, C. Kallin and A.J. Berlinsky, Phys. Rev. B 54, R6897 (1996).

12 S. Haas, A.V. Balatsky, M. Sigrist and T.M. Rice, condmat/9703082.

${ }^{13}$ A.A. Abrikosov and L.P. Gorkov, Sov. Phys. JETP 12, 1243 (1961).

14 S. Dukan and Z. Tesanovic, Phys. Rev. B 56, 838 (1997).

15 Z. Gedik and Z. Tesanovic, Phys. Rev. B 52, 527 (1995).

${ }^{16}$ P.G. de Gennes, Superconductivity of Metals and Alloys (Addison-Wesley, Reading, MA, 1989).

17 V.G. Kogan, J. Low Temp. Phys. 20, 103 (1975).

${ }^{18}$ Y.A. Bychkov and E.I. Rashba, Sov. Phys. JETP 58, 1062 (1983). 